"Proserpinens Park"

Goethes Wahlverwandtschaften als Selbstkritik der Moderne 
Giovanni Sampaolo

\section{»Proserpinens Park«}

Goethes Wahlverwandtschaften als Selbstkritik der Moderne

Aus dem Italienischen von Annette Kopetzki

Verlag J.B. Metzler

Stuttgart - Weimar 
Übersetzung und Publikation wurden gefördert durch die folgenden Institutionen:

Italienisches Außenministerium

Università degli Studi »G. d'Annunzio«, Chieti/Pescara (Italien)

Titel der Originalausgabe:

Critica del moderno, linguaggi dell'antico. Goethe e Le affinità elettive, Rom: Carocci editore, 1999

Bibliografische Information Der Deutschen Bibliothek

Die Deutsche Bibliothek verzeichnet diese Publikation in der Deutschen Nationalbibliografie; detaillierte bibliografische Daten sind im Internet über $<$ http://dnb.ddb.de $>$ abrufbar.

ISBN 978-3-476-01948-6

ISBN 978-3-476-02927-0 (eBook)

DOI 10.1007/978-3-476-02927-0

Dieses Werk einschließlich aller seiner Teile ist urheberrechtlich geschützt. Jede Verwertung außerhalb der engen Grenzen des Urheberrechtsgesetzes ist ohne Zustimmung des Verlages unzulässig und strafbar. Das gilt insbesondere für Vervielfältigungen, Übersetzungen, Mikroverfilmungen und die Einspeicherung und Verarbeitung in elekronischen Systemen.

(C) 2003 Springer-Verlag GmbH Deutschland

Ursprünglich erschienen bei J.B. Metzlersche Verlagsbuchhandlung

und Carl Ernst Poeschel Verlag GmbH in Stuttgart 2003

www.metzlerverlag.de

info@metzlerverlag.de 
Für Beatrice Cignitti, Malerin 


\section{Inhalt}

Vorwort zur deutschen Ausgabe $\ldots \ldots \ldots \ldots \ldots \ldots \ldots$ IX

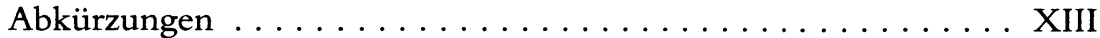

Abbildungsverzeichnis $\ldots \ldots \ldots \ldots \ldots \ldots \ldots \ldots \ldots \ldots$

Einleitung: Das Experiment der Antike in der Moderne. . . . . . . 1

1 Götterbilder und chemische Zersetzung:

Metaphern einer Epoche . . . . . . . . . . . . 1

2 Die Idylle neu gründen: kultivieren

Zur Weimarer Utopie . . . . . . . . . . . . . . 25

3 "Geschichte in Mythologie verwandeln"

Zur Lektüre der Wahlverwandtschaften . . . . . . . . . 43

I Figuren des Kultivierens. Letzte Bilder des Klassischen. . . . . 69

1.1 Charlottes Kultur . . . . . . . . . . . . . . . . . . . 69

1.2 Ottilies Natur . . . . . . . . . . . . . . . . . . . 90

1.3 Das Zerbrechen des Paares als Verlust der menschlichen Ganzheit. . . . . . . . . . . . . . . . . . . . . . 101

1.4 Hoffnung auf einen neuen Anfang: der Name des Erben . . . . 107

2 Die Zeit des Hades. Nach $1806 \ldots \ldots \ldots \ldots \ldots \ldots \ldots$

2.1 Ein umzingelter Frieden . . . . . . . . . . . . . . 125

2.2 Vom Garten zum Park: Der Triumph des Narzißmus. . . . . . 160

3 Der Raub der Ottilie. Verführung als Parabel . . . . . . . . 183

3.1 Die Katastrophe am Seeufer. . . . . . . . . . . . . . . . 183

3.2 Die tragische Wahlverwandtschaft. . . . . . . . . . . . . . 199

4 Klassisch-romantische Mysterien. . . . . . . . . . . . . . . . 219

4.1 Schluß des Romans und romantische Dramenformen . . . . . . 219

4.2 Ein Bildergeflecht: "quod ostendat revisentibus" . . . . . . 238

4.3 Die allegorische Apotheose: Überleben des IIdeals?? . . . . . . . 247

Epilog: $\gg / n$ tausend Formen...« . . . . . . . . . . . . . . . . . 261

Abbildungen . . . . . . . . . . . . . . . . . . . 269

Bibliographie. . . . . . . . . . . . . . . . . . . . . . . 273

Namenregister . . . . . . . . . . . . . . . . . . . . 289

Titelregister . . . . . . . . . . . . . . . . . . . . . . . 295 


\section{Vorwort zur deutschen Ausgabe}

Den Leitfaden dieser Lektüre der Wahlverwandtschaften, Goethes heute wohl meistgelesenem Roman, der in der Forschung wie kein zweiter diskutiert wird, bilden Zielsetzungen und Kursänderungen des Weimarer Klassizismus bei seiner unablässigen Konfrontation mit der entstehenden Moderne. Ein erster Schwerpunkt liegt dabei im Gegensatz von Antike und Moderne, der sich im späten 18. Jahrhundert in der deutschen kulturellen Debatte als verbindliches Deutungsmuster eines in vieler Hinsicht krisenhaften historischen Horizonts etabliert. Das Bildfeld der chemischen 'Scheidungen', bekanntlich ein Eckpfeiler des Romans, erweist sich aus dieser Perspektive und nicht ausschließlich bei Goethe als Figuration der als eine Bedrohung erlebten sozialen und kulturellen Ausdifferenzierung und allgemeinen Labilität in der neuen Zeit. Als leitender Lösungsversuch, an dem der Klassizismus experimentierend arbeitet, zeichnet sich der Aufbau einer an antiken VorBildern orientierten ästhetischen Anthropologie ab. Damit eng verflochten ist die vielfach variierte Anverwandlung einer traditionsreichen bildsprachlichen Semantik von 'Kultur , die von der natürlichen Verankerung jeder Zivilisation in der agri cultura, dem Landbau, ausgeht. Im realen Kontext des Herzogtums Sachsen-Weimar schien ihre Kombination mit utopisch aufgeladenem physiokratischem Gedankengut inmitten der neuen Konstellation, die durch Geldwirtschaft und Revolutionen geprägt war, eine Zeitlang auf einen heilen Zugang zur sozialen Erneuerung hinzuweisen.

Damit sind Diskurse einer ganzen Epoche benannt. Der anspruchsvolle ästhetische Text der europäischen Moderne wird hier aber nicht als belegende Artikulation, sondern eher als Bruchstelle der Diskurse angesehen, das heißt als ein Ort, wo kollektive kulturelle Ordnungen zwar gewiß zum Tragen kommen, aber nur, um gebrochen zu werden. Das soll allerdings nicht bedeuten, daß hier etwa gezielt nach jenen Inkonsequenzen, Entgleisungen und Entropiemomenten der Kommunikation gesucht würde, auf welche die dekonstruktivistische Lektürepraxis der letzten Jahrzehnte uns alle verdienstvoll und mit durchaus sympathischem Freiheitspathos, aber auch mit eigensinniger Einseitigkeit aufmerksam gemacht hat, nämlich ohne Augenmerk auf das sozial formende Moment des Systemischen und Konventionalisierten in kommunikativen Praktiken, das doch die Verständlichkeit der dekonstruktivistischen Kommunikation selbst trägt und ermöglicht. Ganz im Gegenteil: bei der vorliegenden Lektüre ergaben sich Risse und Brüche gerade beim Versuch, die prägnante Kohärenz nachzuvollziehen, die in einem Werk wie Die Wahlverwandtschaften schon auf formaler Ebene erstaunlich konsequent, ja geradezu befremdend realisiert ist. Ins Blickfeld gerieten dabei gerade nicht nahtlose Überfuhrungen zwischen kulturellen 
Strukturen und solchen des Textes, sondern Bruchlinien, die den Roman gleichsam wie Verwerfungen durchziehen, welche die Schichtung der Diskurse und ihr Brüchigwerden traumatisch bloßlegen. So gesehen bilden Die Wahlverwandtschaften wahrhaftig die tragische Idylle einer scheiternden >Poetik der Kultur.

Der Modus, wie Normen im Textgewebe jeweils ausgestellt sind, um verletzt $\mathrm{zu}$ werden, stellt unter solchen Normbrüchen eine 'Familienähnlichkeit (im Sinne Wittgensteins) her, eine sonderbare /Verwandtschaft , die man eine negative Kohärenz nennen könnte. Der eigentliche Gegenstand dieser Untersuchung sind somit die Differenzen zwischen Goethes sich wandelndem Bildsystem und seiner kulturellen Umwelt. Differenzen können aber nur anhand der ihnen zugrundeliegenden Gemeinsamkeiten festgestellt und eingeschätzt werden. In diesem Fall besteht das Gemeinsame und Verbindende vor allem in einem vielschichtigen Netzwerk aus Bildern, Tropen, Mythen, Vorstellungen und /Konstruktionen von Wirklichkeitı, das zu einem guten Teil nicht diskursiv, sondern nur über die Rekonstruktion vielfältiger Bildbezüge zu erkunden ist. Die Rekursivität der historisch gegebenen Bildervorräte und die Rekurrenz der Bildzusammenhänge sowohl in Goethes Euvre als auch in seinem klassizistischen Umfeld können jene schillernde Welt der Zeichen wenigstens bis zu einem gewissen Grad plausibel lesbar machen. In diesem Sinne wurde hier versucht, der Komplexität kulturhistorischer Kontexte wie auch der modellhaften Komplexität ästhetischer Konstruktion gerecht zu werden. Der Blick war auf das konkrete Weltwissen, den Erfahrungsraum des Autors und seiner Zeitgenossen zu richten, ohne das Sinnlich-Bildliche der \Dichtung` zugunsten einer eindeutigen 'Wahrheit‘ aus dem Auge zu verlieren.

Darum waren schon rein pragmatisch Anleihen bei unterschiedlichen heuristischen Verfahrensweisen vonnöten, je nach den Fragen, die der Text aufzuwerfen schien. Diese Anleihen durchkreuzen eklektisch einen literarhermeneutischen Habitus, der sich freilich selbst als entsubstanzialisiert und weitestgehend 'grund-los` versteht, und sollten ihn - wenigstens war dies die Absicht - in einer wechselseitigen Integration bereichern. Themenstellung, Methodenvielfalt, sowie eine zumindest ansatzweise angestrebte Ausweitung der Quellenbasis über die Höhenkammliteratur oder auch generell über die belles-lettres hinaus, decken sich jedoch nur teilweise mit dem weit abgesteckten Rahmen dessen, was seit einigen Jahren unter dem Sammelbegriff `Kulturwissenschaft‘ zur Debatte steht.Vielmehr orientieren sie sich an dem enger gefaßten Entwurf einer Literaturwissenschaft, die ihre eigenen Positionen hinterfragt, sich mit kulturwissenschaftlichen Problemstellungen befaßt und nach entsprechenden Instrumenten sucht. Von Literatur ist hier also hauptsächlich die Rede, denn allein das ästhetische Faszinosum hat - das sei hier in aller unzeitgemäßen Evidenz eingestanden - eine Erkenntnisbemühung angetrieben, die doch nicht umhin konnte, 
über die Literatur hinweg auf benachbarte und entferntere Felder zu gelangen, mit denen die Literatur stets in einem regen Austauschverhältnis steht.

Zum Aufbau des Buches: Die Einleitung skizziert den allgemeinen Bezugsrahmen. Deshalb wird dort an viel Bekanntes erinnert, doch wird Bekanntes zugleich auf Neues hin perspektiviert, denn jeder Satz der Einleitung bereitet einen Schritt der Interpretation vor. Dies ist nicht nur als eine Einstiegserleichterung für den Laien gemeint, sondern auch und vor allem als Entlastung der folgenden Kapitel, damit sie Bezüge voraussetzen können, die in der Einleitung zusammenhängend dargelegt werden. Methodisches wird dort anhand einer kurzen Diskussion der neueren Forschung zu Goethes Roman ebenso kurz erörtert. Die allgemeinsten Themen der Kapitel 1 bis 3 wurden eingangs angedeutet. Kapitel 4 hat Goethes Auseinandersetzung mit der betont 'modernistischen Ästhetik der Romantik zum Gegenstand. Hier wird unter einem ganz besonderen Blickwinkel nämlich dem der deutschen Calderón-Rezeption im frühen 19. Jahrhundert - dargelegt, wie Goethe zu einer spannungsvollen Synthese zwischen den tragisch einander gegenüberstehenden Optionen der naturhaften Antike und der künstlichen Moderne findet. So kommt es nicht einfach zu einem die Tragik mildernden Nachspiel am Romanende. Denn Goethe revidiert in diesem Zusammenhang zentrale Konzeptionen seiner Weltsicht in Richtung auf Alterität. Über die Krise des Klassizismus, die Die Wahlverwandtschaften ausloten und austragen, geht ein Gedicht aus dem West-östlichen Divan hinaus, das noch deutlicher im Zeichen dieser Öffnung für Pluralisierung steht - sowohl des Menschlichen als auch der Natur und der Zeichen, durch die Erkenntnis aufgebaut wird. Es wird im Epilog als Fallbeispiel der Fortführung dieses Weges im Spätwerk herangezogen.

Entstanden ist diese Studie zunächst zwischen 1995 und 1998 als Doktorarbeit an der Universität Pisa. Prof. Luciano Zagari (Pisa) und Prof. Giuliano Baioni (Venedig), die sie während dieser Phase betreut haben, gilt noch einmal mein erster Dank. Die Buchfassung erschien dann 1999 in Rom. Aus dem Zustandekommen der vorliegenden deutschen Ausgabe ergab sich die Gelegenheit, das Ganze selektiv zu aktualisieren und vor allem an vielen Stellen ergänzend zu überarbeiten. Daraus ist eine insgesamt erweiterte und - in den Grenzen einer schon bestehenden Struktur - überdachte Neufassung geworden. Der Übersetzerin, Dr. Annette Kopetzki, die diesen Umwandlungsprozeß geduldig mitgemacht hat, sei an dieser Stelle gedankt.Vielen bin ich aber zu Dank verpflichtet. Allen voran Prof. Francesco Fiorentino (Rom) und Prof. Vanda Perretta (Rom) für die kritische, nachfragende Lektüre vieler Vorarbeiten. Für Hinweise und Ermunterung danke ich Prof. Eugenio Bernardi (Venedig), Dr. Ursula Bongaerts (Rom), Prof. Giovanna Cermelli (L'Aquila), Prof. Paolo Chiarini (Rom), Prof. Luca Crescenzi (Pisa), Prof. Franco Cuccurullo (Chieti), Prof. Alberto Destro 
(Bologna), Prof. Giuseppe Dolei (Catania), Prof. Maria Carolina Foi (Triest), Prof. Marino Freschi (Rom), Dr. phil. habil. Jochen Golz (Weimar), Prof. Christoph Jamme (Lüneburg), Prof. Helmut Koopmann (Augsburg), Prof. Francesco Marroni (Pescara), Prof. Ida Porena (Rom), Prof. Aldo Venturelli (Urbino) und Prof. Roberto Venuti (Siena) - ganz besonders aber Prof. Heinz Schlaffer (Stuttgart). Zwischen den Stationen dieser Recherche in Göttingen, Marbach, Weimar, Stuttgart, München und Berlin wurden dem Auslandsgermanisten in seiner Heimatstadt die Bestände des Istituto Italiano di Studi Germanici immer besonders leicht zugänglich gemacht, wofür ich Dr. Bruno Berni sehr verbunden bin. Dasselbe gilt für die Casa di Goethe "al Corso" und deren Mitarbeiterinnen Frau Renata Crea und Frau Dorothee Hock. Einem Aufsatz von Prof. Franco D'Intino (Perugia) zum Persephone-Motiv bei Leopardi (I misteri di Silvia, in: Filologia e Critica, XIX, 1994, S. 211-271) verdanke ich Anregungen, die den Boden für die Konzeption dieses Buches entscheidend vorbereiteten. Frau Grazia Aquilino und Prof. Maurizio Cignitti danke ich dafür, daß sie sich in diesem Zeitraum mehr denn je meiner Tochter Agnese gewidmet haben; Dr. Bernd Lutz und Dr. Oliver Schütze vom Verlag für die freundliche Aufnahme ins Programm und die schöne Ausstattung des Bandes.

Rom, im September 2003

G.S. 


\section{Abkürzungen}

\section{Schriften von Johann Wolfgang Goethe}

FA

GA

HA

HAB

MA

WA

DVjs

DW

EG

GG

$\mathrm{GJb}$

Goethes Bibliothek

GR

GRWV
Sämtliche Werke. Briefe, Tagebücher und Gespräche, hg. v. Fr. Apel u.a., Frankfurt/M. 1985-1999 (Frankfurter Ausgabe)

Gedenkausgabe der Werke, Briefe und Gespräche, hg. v. E. Beutler, Zürich 1948-1960

Werke. Hamburger Ausgabe in 14 Bänden, hg. v. E. Trunz, durchges. Ausg., München 1988

Briefe, hg. v. K.R. Mandelkow, München $1988^{4}$ (Goethes Briefe. Hamburger Ausgabe)

Sämtliche Werke nach Epochen seines Schaffens, hg. v. K. Richter u.a., München 1985-1999 (Münchner Ausgabe) Goethes Werke, hg. im Auftrage der Großherzogin Sophie v. Sachsen, Weimar 1887-1914, Nachdr. München 1987 und Stuttgart/Weimar 1999 (Weimarer Ausgabe)

\section{Weitere Abkürzungen}

Deutsche Vierteljahrsschrift für Literaturwissenschaft und Geistesgeschichte

"Die Wahlverwandtschaften". Eine Dokumentation der Wirkung von Goethes Roman 1808-1832, hg. v. H. Härtl, Berlin 1983

Eckermann, J.P., Gespräche mit Goethe in den letzten Jahren seines Lebens (1836-48), München $1988^{3}$

Goethes Gespräche in vier Bänden, auf Grund der Ausgabe und des Nachlasses von F. Frhrn. v. Biedermann ergänzt und hg. v. W. Herwig, Zürich-Stuttgart 1969

Goethe-Jahrbuch

Goethes Bibliothek. Katalog, bearb. v. H. Ruppert, Weimar 1958

Goethe und die Romantik. Briefe mit Erläuterungen, hg. v. O. Walzel/C. Schüddekopf, Weimar 1898-1899 (Schriften der Goethe-Gesellschaft, Bde. XIII-XIV), Nachdr. Leipzig 1984

Rösch, E. (Hg.), Goethes Roman "Die Wahlverwandtschaften ", Darmstadt 1975 
JbDSG Jahrbuch der Deutschen Schiller-Gesellschaft

JbFDH Jahrbuch des Freien Deutschen Hochstifts

KFSA Kritische Friedrich-Schlegel-Ausgabe, hg. v. E. Behler u.a., Paderborn/München/Wien 1958ff

NA Schillers Werke. Nationalausgabe, hg. v. J. Petersen u.a., Weimar 1943ff

PEGS Publications of the English Goethe Society

Bei den bibliographischen Hinweisen in den Anmerkungen bezeichnen die römischen Zahlen den Band, die arabischen Ziffern die Seiten. Bei Hinweisen auf WA und FA bezeichnet die erste römische Zahl die jeweilige Abteilung.

Goethes Werke werden so weit wie möglich aus HA (der verbreitetsten Ausgabe) zitiert. Die Wahlverwandtschaften werden aus HA VI, 242-490 zitiert, im fortlaufenden Text beziehen sich die Seitenzahlen in Klammern auf diese Ausgabe.

Um dem Leser die Orientierung in der Chronologie der Werke zu erleichtern, wird in den meisten Fällen bei dem ersten Hinweis auf einen Text in Klammern das Erscheinungsjahr oder das Jahr der letzten Fassung genannt.

In allen Fällen, in denen auf den bibliographischen Hinweis nicht die Bemerkung »Hervorhebung i.O." folgt, wurden kursive Hervorhebungen, falls vorhanden, vom Verfasser eingefuhrt, um besondere Aufmerksamkeit auf die Ausdrücke zu lenken, die jeweils im Zentrum der Untersuchung stehen. 


\section{Abbildungsverzeichnis}

Abb. 1 und 2: Ceres erklärt der wilden Menschheit den Anbau des Getreides/ Ceres führt die gerade zivilisierten Menschen zur Anbetung des Zeus. Nach J.M. Wagner (Stiche von F. Ruscheweyh).

Aus: Das eleusische Fest. Schillers Dichtung bildlich dargestellt von I.M. Wagner, Stuttgart/Tübingen: Cotta, 1817, Kupfer Nr. 3 und 20 (zu S. 39).

Abb. 3: J.W. Goethe, Proserpina (Bleistiftzeichung, um 1787/88).

Nach: Corpus der Goethe-Zeichnungen, bearb. v. G. Femmel, Bd. III, Leipzig 1965, Nr. 156 (zu S. 96).

Abb. 4: $\quad$ Pluto (Stich nach Karsten).

Nach: K.Ph. Moritz, Götterlehre (1790), in: ders., Werke, hg. v. H. Günther, Frankfurt/M. 1981, Bd. II, S. 835 (zu S. 120).

Abb. 5: J.Fr. Anthing, Goethes Silhouette (Tusche, Wasserfarben und in Kupfer gestochenes Selbstbildnis). Mit Goethes Eintrag vom 7. September 1789 im Stammbuch des Silhouetteurs.

Nach: Insel-Almanach auf das Jahr 1960, nach S. 130 (zu S. 181).

Abb. 6: Der Raub der Proserpina (Stich nach einer Hamiltonschen Vase).

Aus: A.L. Hirt, Bilderbuch für Mythologie, Archäologie und Kunst, Bd I: Die Tempelgötter, Berlin 1805, S. 72, Abb. 22 (zu S. 194). 